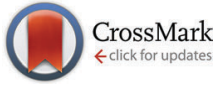

Cite this: Chem. Commun., 2016, 52,5961

Received 16th February 2016, Accepted 23rd March 2016

DOI: $10.1039 / c 6 c c 01434 h$

www.rsc.org/chemcomm

\section{Microfluidic generation of encapsulated droplet interface bilayer networks (multisomes) and their use as cell-like reactors $\dagger$}

\author{
Yuval Elani, ${ }^{\star a b}$ Xavier C. I. Solvas, $\ddagger^{a}$ Joshua B. Edel, ${ }^{a b}$ Robert V. Law ${ }^{a b}$ and \\ Oscar Ces*ab
}

Compartmentalised structures based on droplet interface bilayers (DIBs), including multisomes and compartmentalised vesicles, are seen by many as the next generation of biomimetic soft matter devices. Herein, we outline a microfluidic approach for the construction of miniaturised multisomes of $\mathrm{pL}$ volumes in high-throughput and demonstrate their potential as vehicles for in situ chemical synthesis.

Droplet interface bilayers (DIBs) are lipid membranes which are formed when two lipid monolayer-coated water-in-oil droplets are brought into contact. ${ }^{1}$ Oil is excluded from the interface and a bilayer is formed. Critically, when three or more droplets are joined together, a bilayer network can be generated. ${ }^{1-4}$ DIBs are emerging as a new platform for the construction of soft matter devices with incorporated biological functional components, including protein pores (both natural and engineered), enzymes, DNA, protein synthesis machinery and polymerisable lipids. ${ }^{1,5-8}$ In this sense DIB devices can be considered to be a sub-discipline in bottom-up synthetic biology, where functional cell-like structures are constructed using simple chemical and biological building blocks. To date, the power of DIB devices for a range of applications has been demonstrated. They have been shown to be capable of mimicking simple electrical circuits and logic gates, ${ }^{3,9}$ acting as biobatteries, and harvesting light for autonomous energy generation. ${ }^{1,3,4,9}$ They have been engineered to be responsive to their environment, ${ }^{7}$ and as tools to screen ion channel blockers, ${ }^{10}$ to study the effects of membrane asymmetry,${ }^{11}$ and to dynamically control the concentration of embedded proteins on a membrane patch. ${ }^{12}$ Extended 3D networks can be constructed using droplet microfluidics ${ }^{2,13,14}$

\footnotetext{
${ }^{a}$ Department of Chemistry, Imperial College London, Exhibition Road, London SW7 2AZ, UK. E-mail: yuval.elani10@imperial.ac.uk, o.ces@imperial.ac.uk ${ }^{b}$ Institute of Chemical Biology, Exhibition Road, London SW7 2AK, UK $\dagger$ Electronic supplementary information (ESI) available: Experimental; device design and fabrication; video of on-chip multisome generation. See DOI: 10.1039/ c6cc01434h

\# Current address: Institute for Chemical and Bioengineering, Department of Chemistry and Applied Biosciences, ETH Zürich, CH-8093, Switzerland.
}

and droplet printing, ${ }^{15}$ and have been engineered to show cooperative behaviours and possess tissue-like features. ${ }^{15}$

One inherent feature of DIBs is that they exist in a bulk oil environment. This prevents their use in physiological (i.e. aqueous) environments and has proved to be a bottleneck for the use of DIBs for a range of potentially powerful applications, for example, as compartmentalised drug delivery machines ${ }^{16}$ or as biosensors. To address this, Villar et al. encapsulated several lipid-stabilised water droplets in a larger oil-in-water droplet, allowing a droplet network to operate in an aqueous environment. ${ }^{17}$ These structures, known as multisomes, were shown to respond to both $\mathrm{pH}$ and temperature and could have protein pores reconstituted in their membranes. Similarly, we have previously generated analogous bilayer networks that do not contain an intermediate oil phase, in the form of multicompartment vesicles. ${ }^{18,19}$ The use of these vesicles as spatially segregated reactors that mimic cellular signalling cascades was later shown. ${ }^{20}$

The major limitation of both these structures arises from how they are generated: they are constructed by manually pipetting individual droplets one-by-one. Droplet sizes are therefore restricted by the minimum pipetting volume, and are thus in the $\mu \mathrm{L}$ volume regime. In addition, each unit has to be assembled manually and individually, leading to a very low throughput of formation, on the order of minutes per single structure. This drastically limits their future applications. For compartmentalised membrane systems to be used as artificial cells, or as soft-matter devices operating in physiological environments, their characteristic dimensions need to be reduced, and their generation throughput increased.

In this communication, we turn to droplet-based microfluidics ${ }^{21}$ to provide a solution to this and to relieve the bottleneck. We design and fabricate devices that generate multisomes, which are an order of magnitude smaller (ca. $4 \mathrm{pL})$ and crucially, with a generation throughput that is three orders of magnitude greater (hundreds of multisomes generated per minute). We can also control the number of droplets in the encapsulated network as well as their content. We prove that there is a bilayer present via a 
A

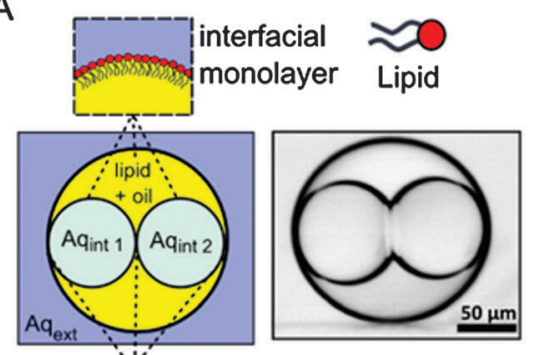

diffusion assay, and demonstrate the potential of the structures as in situ drug synthesis machines by synthesising a pharmacologically relevant chemical moiety in their interior.

To generate multisomes we fabricated a device that consisted of two sequential droplet generation modules separated by a meandering channel (Fig. 1 and ESI, $\dagger$ Video). The first junction consisted of two opposing T-junctions with two different aqueous inlets, yielding water-in-oil (w/o) droplets of alternating compositions. $25 \mathrm{mg} \mathrm{ml}^{-1}$ of diphytanoylphosphatidylcholine (DPhPC) lipid was dissolved in the oil phase, which self-assembled as a monolayer around the droplets as they travelled through the meander. This enabled bilayers to form at the droplet-droplet interfaces, and prevented droplet coalescence upon encountering the outer aqueous phase.

The second junction contained a flow-focusing channel geometry, which encapsulated a set number of aqueous droplets in larger oil droplets, producing double emulsion based multisomes. This was followed by additional meandering and channel widening where droplets were stored and monitored.
DIBs were formed between the multiple inner droplets themselves, and between the inner droplets and the external aqueous phase. Multisome dimensions and architecture were predominately determined by the device geometry, with the inner aqueous droplet and external oil droplet diameters regulated by the characteristic dimensions of the first and second flow focusing junctions respectively. Flow rates are a second-order determinant of droplet size, with smaller droplets produced by increasing the continuous phase flow. ${ }^{22}$ Likewise, flow rates of the segmented phase affect droplet generation frequencies. By increasing the frequency at which the inner droplets were generated compared to the outer droplets, multisomes with a larger number of innercompartments could be produced on the same device (see the ESI, $†$ Video). Droplet encapsulation number could thus be userdefined, yielding multisomes with varying numbers of inner droplets (between one and four, Fig. 1C).

Multisomes were generated at a rate of 300 per minute and stored in channels (Fig. 1D). 95\% encapsulation efficiency was achieved; however, the emulsion survival rate stood at 
$88 \%(n=300)$ for two-compartment multisomes, with a proportion of droplet pairs merging shortly after formation. If individual droplets were still intact after 30 seconds, they were stable for the course of the experiments (60 minutes). An important factor in multisome stability was the degree of confinement of the inner droplets. Higher confinement led droplets to squeeze against each other, exerting forces which increased the probability of bilayer rupture. As three- and four-compartment multisomes are inherently more confined, their survival rates were correspondingly lower. Changing device geometry to decrease the confinement (for example, to produce a larger intermediate oil droplet) would be necessary to produce stable multisomes with more internal compartments.

Average inner and outer droplet volumes were $1.56 \mathrm{pL}(72 \mu \mathrm{m}$ radius) and $8.01 \mathrm{pL}(128 \mu \mathrm{m}$ radius) respectively, with the corresponding coefficients of variation of $3.4 \%$ and $1.5 \%$ (Fig. 1E). Droplet volumes were therefore highly uniform, a common feature associated with droplet-based microfluidic technologies. The use of microfluidics therefore enables the generation of compartmentalised membrane systems with finer size resolution and an order of magnitude smaller volumes than can be reached with pipetting. Further miniaturisation will allow cell-sized dimensions to be reached (and surpassed), paving the way for drug-delivery applications.

We then demonstrated the ability of multisomes to be used as multi-compartment chemical microreactors via the synthesis of a biologically relevant compound (Experimental details available in the ESI $\dagger$ ). We constructed multisomes consisting of two inner droplets, which acted as modules in the chemical reactor: one droplet acted as a chemical reservoir and the other as a reaction chamber. We produced multisomes with two different cargoes contained in each of the encapsulated droplets. In one droplet, a membrane impermeable fluorogenic pyrylium compound (Chromeo P540) ${ }^{23}$ was encapsulated, and in the other a membrane permeable primary amine (ethanolamine, $\mathrm{HOCH}_{2} \mathrm{CH}_{2} \mathrm{NH}_{2}$ ). The amine-containing droplet acted as a reservoir, which steadily fed the amine through the DIB into the adjacent droplet, in which a chemical transformation took place (Fig. 2). Ethanolamine was able to gradually diffuse through the bilayer due to its neutral charge and small size $(\log P=-1.31) .{ }^{24}$ As the amine entered the adjacent compartment, it reacted with pyrylium to yield a pyridinium salt, which was fluorescent (ex $=533 \mathrm{~nm}$, em $=627 \mathrm{~nm}$, see $\mathrm{ESI} \dagger$ ). A fluorescence increase in the adjacent compartment over time was observed (Fig. 2B), with a gradual increase over a period of $800 \mathrm{~s}$ before a plateau in fluorescence intensity as the reagent was depleted. Fluorescence was not seen to decrease from its maxima, and the surroundings exhibited no observable fluorescence increase. This suggests that the reaction product does not leak into the oil or external water phase over the time course of the experiment, most likely a result of the pyridinium positive charge prohibiting translocation across the bilayer or partitioning into the oil phase.

Previous studies show that the rate determining step for the reaction between pyrylium and analogous primary amines is the intermediate ring closure to yield the pyridinium product $\left(k=4.04 \times 10^{-3} \mathrm{~s}^{-1}\right) .{ }^{25}$ The corresponding approximate reaction
A
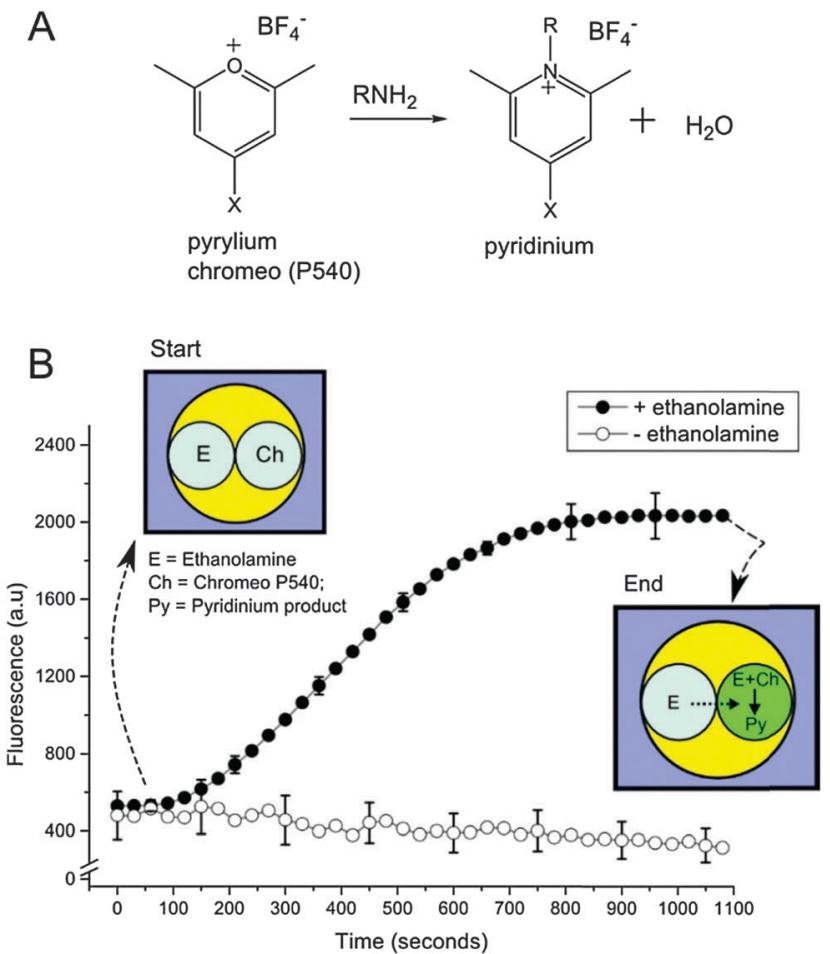

C
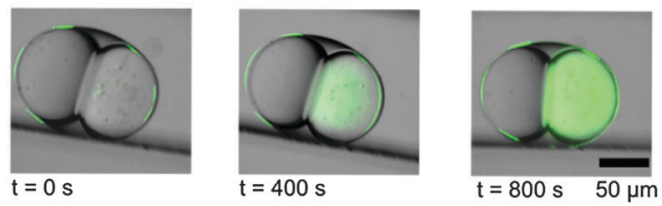

Fig. 2 Multisomes as reactors for on-board synthesis. (A) Reaction scheme between pyrylium and a primary amine, yielding fluorescent pyridinium. (B) Fluorescence intensity of the pyrylium-containing compartment over time. Error bars $=$ one standard deviation, $n=5$. The two reagents were originally isolated in the two compartments. As ethanolamine diffused through the DIB, fluorescent pyridinium product was formed. (C) Fluorescence/brightfield composite image of the reactor at start, middle, and endpoints of experiment, showing gradual reactant diffusion and reaction progression. Brightness and contrasts enhanced equally across all images.

completion timescales $(\sim 750 \mathrm{~s})$ are comparable to the ones we observe $(\sim 810 \mathrm{~s})$. The fact that reaction times were not significantly slower in our system indicates that the rate of ring closure is comparable to, or faster than, diffusion across the membrane.

In control experiments, identical multisomes were generated but with no ethanolamine present. No fluorescence increase was seen, showing that the reaction was not occurring due to external sources of primary amine (originating, for example, from the device fabrication process).

Together with the lack of fluorescence increase in the absence of a bilayer, these experiments proved the existence of a DIB. In addition, the results establish the applicability of such systems for in situ drug synthesis, as the pyridinium reaction products and their analogues are found in a wide range of medicinal and agricultural products, including anaesthetics, antiprotozoals, and antiallergenics. $^{26,27}$ Their bioactivity and commercial use therefore 
made them attractive candidates to be used as a proof-of-concept for our system.

Although the above experiment demonstrates the use of multisomes for simple one-step reactions, by adding more compartments this can be extended to multi-step chemical reactions, each step being performed in distinct compartments. ${ }^{20}$ Furthermore, the fact that it is possible to tune droplet sizes using simple microfluidic engineering principles, and that droplet sizes in turn determine bilayer area, raises the possibility of modulating reaction kinetics using the architecture of the system.

These results demonstrate a new class of biomimetic microreactors bearing advantages associated with subcompartmentalisation. Compartmentalisation allows isolated specialised modules to be introduced, with more elaborate operations introduced in the future, including waste droplets and processing elements, allowing for greater complexity than traditional uni-compartment systems. The system's modular nature also allows for multiple potentially interfering processes (non-orthogonal reactions) to occur in a single droplet collection by segregating them in space.

The presence of lipid bilayers between compartments renders the multisomes generated here as distinct entities to conventional double emulsions. ${ }^{28,29}$ Crucially, they provide an interface through which reagent transfer can take place, and increase the biomimetic potential of the structures enabling them to be used for synthetic biology and biotechnology applications. The use of membranes to delineate compartments allows functionalization by biological components including transmembrane proteins, mechanosensitive channels, and antibodies, as well as ensuring biocompatibility. It also enables researchers to take advantage of the varied biophysical behaviours of membranes, and to exploit them to modulate processes-such as pore formation with a change in temperature, electromagnetic radiation, or in response to chemical stimulus - to yield smart and responsive features. The above attributes provide an opportunity for the design of dormant systems, which become active upon encountering set stimuli, with applications for in vivo drug synthesis and delivery.

In conclusion by leveraging microfluidic technologies we were able to scale-up the generation throughput of multisomes to hundreds a minute, and scale down their size to the $\mathrm{pL}$ regime, all while maintaining control over the number of droplets in the encapsulated network and their content. This allows the power and potential of droplet networks to be extended to an aqueous setting. We also demonstrate the use of multisomes generated via microfluidics as a platform to perform rudimentary synthetic chemistry in a cell-like device, paving the way for the generation of truly functional and responsive compartmentalised softmatter devices for a range of applications, including in situ drug synthesis and as self-contained diagnostic devices.
Microfluidic technologies such as these could be used as an enabling technology for synthetic biology, facilitating scale up in generation throughput and scale down in size that is needed for them to see functional use.

This work was supported by the EPSRC via grants EP/J017566/1 and EP/K038648/1, and by a Centre for Doctoral Training Studentship from the Institute of Chemical Biology (Imperial College London) and EPSRC Doctoral Prize Fellowship awarded to YE.

\section{Notes and references}

1 H. Bayley, B. Cronin, A. Heron, M. A. Holden, W. L. Hwang, R. Syeda, J. Thompson and M. I. Wallace, Mol. BioSyst., 2008, 4, 1191-1208.

2 Y. Elani, A. deMello, X. Niu and O. Ces, Lab Chip, 2012, 12, 3514-3520.

3 M. A. Holden, D. Needham and H. Bayley, J. Am. Chem. Soc., 2007, 129, 8650-8655.

4 G. Maglia, A. J. Heron, W. L. Hwang, M. A. Holden, E. Mikhailova, Q. Li, S. Cheley and H. Bayley, Nat. Nanotechnol., 2009, 4, 437-440.

5 H. M. Barriga, P. Booth, S. Haylock, R. Bazin, R. H. Templer and O. Ces, J. R. Soc., Interface, 2014, 11, 20140404.

6 W. L. Hwang, J. Am. Chem. Soc., 2008, 130, 5878.

7 S. Punnamaraju, H. You and A. Steckl, Langmuir, 2012, 28, 7657-7664.

8 M. S. Friddin, H. Morgan and M. R. de Planque, Biomicrofluidics, 2013, 7, 014108.

9 W. L. Hwang, M. A. Holden, S. White and H. Bayley, J. Am. Chem. Soc., 2007, 129, 11854-11864.

10 R. Syeda, M. A. Holden, W. L. Hwang and H. Bayley, J. Am. Chem. Soc., 2008, 130, 15543-15548.

11 W. L. Hwang, M. Chen, B. D. Cronin, M. A. Holden and H. Bayley, J. Am. Chem. Soc., 2008, 130, 5878-5879.

12 L. C. Gross, O. K. Castell and M. I. Wallace, Nano Lett., 2011, 11, 3324-3328.

13 P. Carreras, Y. Elani, R. Law, N. Brooks, J. Seddon and O. Ces, Biomicrofluidics, 2015, 9, 064121.

14 C. E. Stanley, K. S. Elvira, X. Z. Niu, A. D. Gee, O. Ces, J. B. Edel and A. J. deMello, Chem. Commun., 2010, 46, 1620.

15 G. Villar, A. D. Graham and H. Bayley, Science, 2013, 340, 48-52.

16 Y. Elani, R. V. Law and O. Ces, Ther. Delivery, 2015, 6, 541-543.

17 G. Villar, A. J. Heron and H. Bayley, Nat. Nanotechnol., 2011, 6, 803-808.

18 Y. Elani, A. Gee, R. V. Law and O. Ces, Chem. Sci., 2013, 4, 3332-3338.

19 Y. Elani, R. V. Law and O. Ces, Phys. Chem. Chem. Phys., 2015, 17, 15534-15537.

20 Y. Elani, R. V. Law and O. Ces, Nat. Commun., 2014, 5, 5305.

21 X. Casadevall i Solvas and A. deMello, Chem. Commun., 2011, 47, 1936-1942.

22 T. Ward, M. Faivre, M. Abkarian and H. A. Stone, Electrophoresis, 2005, 26, 3716-3724.

23 D. B. Craig, B. K. Wetzl, A. Duerkop and O. S. Wolfbeis, Electrophoresis, 2005, 26, 2208-2213.

24 C. Hansch, A. Leo, D. Hoekman and S. R. Heller, Exploring Qsar, American Chemical Society, Washington, DC, 1995.

25 A. R. Katritzky, J. L. Mokrosz and M. De Rosa, J. Chem. Soc., Perkin Trans. 2, 1984, 849-855.

26 P. Madaan and V. K. Tyagi, J. Oleo Sci., 2008, 57, 197-215.

27 E. F. Scriven and R. Murugan, Pyridine and pyridine derivatives, in Kirk-Othmer Encyclopedia of Chemical Technology, ed. Mary Howe-Grant and executive ed. Jacqueline I. Kroschwitz, 4th edn, 1996.

28 A. Y. Khan, S. Talegaonkar, Z. Iqbal, F. J. Ahmed and R. K. Khar, Curr. Drug Delivery, 2006, 3, 429-443.

29 R. K. Shah, H. C. Shum, A. C. Rowat, D. Lee, J. J. Agresti, A. S. Utada, L.-Y. Chu, J.-W. Kim, A. Fernandez-Nieves and C. J. Martinez, Mater. Today, 2008, 11, 18-27. 